\title{
Preparation of highly multiplexed small RNA sequencing libraries
}

\author{
Helena Persson ${ }^{1}$, Rolf Søkilde ${ }^{1,2}$, Anna Chiara Pirona ${ }^{1, \dagger}$, and Carlos Rovira ${ }^{1,2}$ \\ ${ }^{1}$ Lund University Cancer Center, Faculty of Medicine, Department of Clinical Sciences Lund, \\ Oncology and Pathology, Lund, Sweden and 'BioCARE, Strategic Cancer Research Program, \\ Lund, Sweden
}

tPresent address: German Cancer Research Center (DKFZ), Functional Genome Analysis, Heidelberg, Germany

BioTechniques 63:57-64 (August 2017) doi 10.2144/000114574

Keywords: sequencing; microRNA; non-coding RNA; biomarker

Supplementary material for this article is available at www.BioTechniques.com/article/114574.

MicroRNAs (miRNAs) are 22-nucleotide-long small non-coding RNAs that regulate the expression of protein-coding genes by base pairing to partially complementary target sites, preferentially located in the $3^{\prime}$ untranslated region (UTR) of target mRNAs. The expression and function of miRNAs have been extensively studied in human disease, as well as the possibility of using these molecules as biomarkers for prognostication and treatment guidance. To identify and validate miRNAs as biomarkers, their expression must be screened in large collections of patient samples. Here, we develop a scalable protocol for the rapid and economical preparation of a large number of small RNA sequencing libraries using dual indexing for multiplexing. Combined with the use of off-the-shelf reagents, more samples can be sequenced simultaneously on large-scale sequencing platforms at a considerably lower cost per sample. Sample preparation is simplified by pooling libraries prior to gel purification, which allows for the selection of a narrow size range while minimizing sample variation. A comparison with publicly available data from benchmarking of miRNA analysis platforms showed that this method captures absolute and differential expression as effectively as commercially available alternatives.

MicroRNAs (miRNAs) are a large class of 22 -nucleotide-long small non-coding RNAs that have been identified in diverse domains of life, including animals, plants, and viruses. They are produced from longer, single-stranded RNA precursors possessing a characteristic hairpin structure through sequential processing by the RNase type III endonucleases DROSHA and DICER. Mature miRNAs associate with proteins of the ARGONAUTE family and regulate the expression of protein-coding genes by base pairing to partially complementary target sites preferentially located in the $3^{\prime}$ untranslated region (UTR) of the mRNA (1). Binding by miRNAs interferes with protein production and mRNA stability through mechanisms including translational repression as well as mRNA sequestration and degradation (2).

The expression and function of miRNAs have been extensively studied in the context of human diseases, including cardiovascular disease (3), infections (4), and a large number of neoplasms (5). The possible use of miRNAs as biomarkers for prognostication and treatment prediction has received much attention in the past few years, especially with the hope that miRNAs in the blood could be used as non-invasive biomarkers. For biomarker development, large collections of patient material and appropriate methods of study are needed. After early studies focusing on miRNA discovery using bacterial cloning and Sanger sequencing (6-8), numerous methods have been developed for

\section{METHOD SUMMARY}

Here, we present a rapid, economical, and scalable protocol for preparing highly multiplexed small RNA sequencing libraries that combines off-the-shelf reagents, dual indexing, and sample pooling before gel purification to produce results comparable to commercially available kits at a considerably lower cost per sample. 
expression profiling of known miRNAs using microarrays and real-time qRT-PCR, as well as Nanostring technology. Today, high-throughput sequencing is typically the method of choice since it combines quantitative measurement of miRNA expression with the possibility of discovering new miRNAs (9) as well as detecting miRNA modifications (10). A handful of commercial kits are available for preparing small RNA sequencing libraries, but these typically only allow the simultaneous multiplexing of 24-48 samples and are intended for sequencing on smaller-scale platforms, such as the MiSeq from Illumina. For sequencing larger collections of patient samples, these kits incur unnecessarily high per-sample costs for library preparation and sequencing. They also typically recommend size selection by gel purification of individual samples, a labor-intensive procedure with substantial risk of introducing sampleto-sample variation. One strategy for increasing sample throughput for SOLiD sequencing of small RNAs by dual indexing has been suggested by Tu et al. (11).

Here, we developed a scalable protocol for rapid and economical preparation of a large number of small RNA sequencing libraries in parallel that is based on currently used methods (e.g., kits from Illumina and New England Biolabs), but uses dual indexing for multiplexing beyond 24-48 samples. Together with the use of off-the-shelf reagents, a large number of samples can be sequenced simultaneously on platforms such as the NextSeq or HiSeq from Illumina at a considerably lower cost per sample. The protocol further simplifies sample preparation by pooling libraries prior to gel purification, which allows selection of a narrow size range while minimizing sample variation. Our protocol can also easily be extended to further increase the number of libraries prepared and sequenced simultaneously, or it can be used for the preparation of small sample numbers, with or without library pooling.

\section{Materials and methods}

For ligation and size-selection tests, the SK-BR-3 breast cancer cell line

A
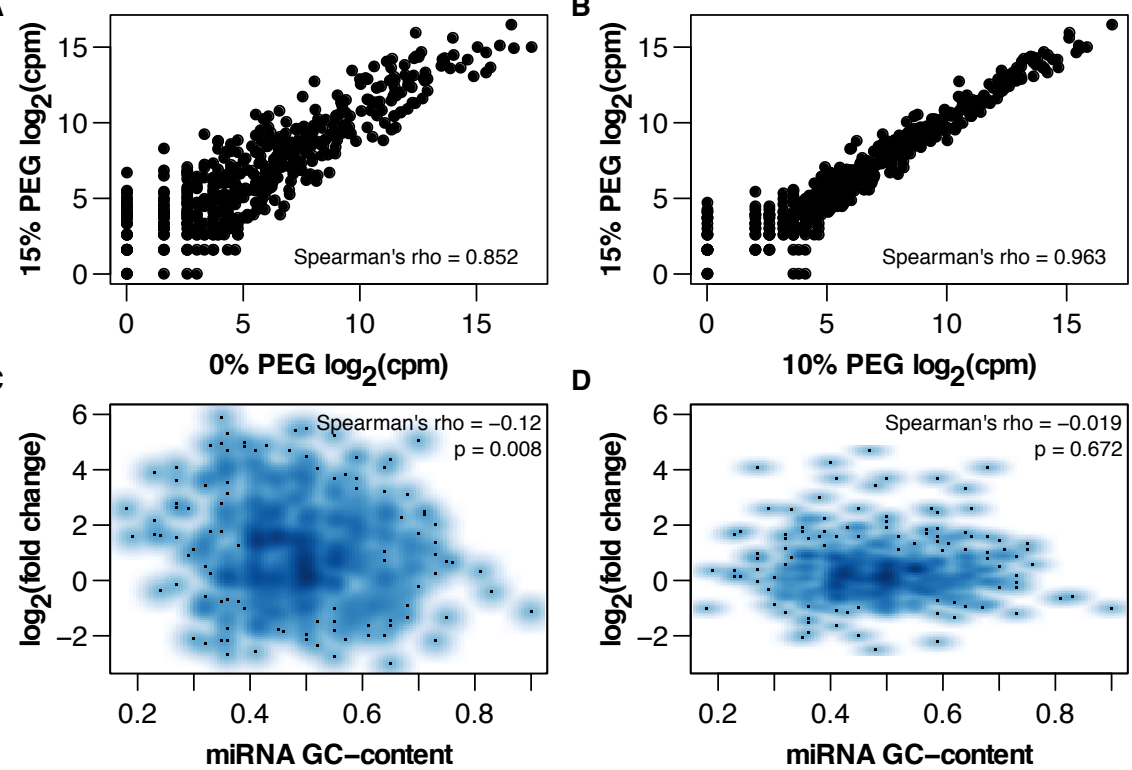

E

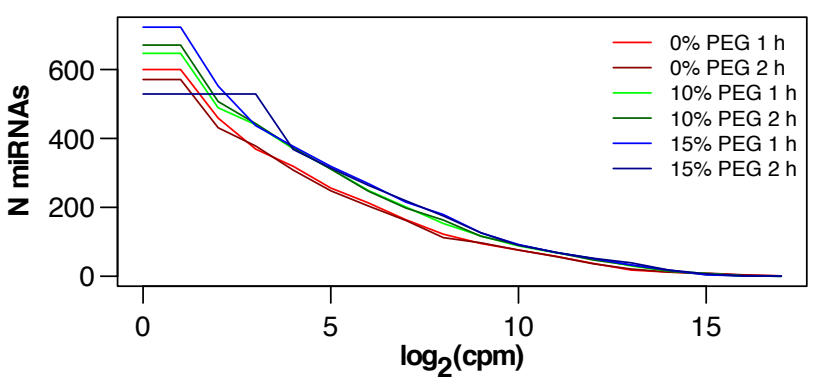

Figure 1. Increasing adapter ligation efficiency through addition of polyethylene glycol (PEG) reduces small RNA sequencing library composition bias. The presence of PEG during RNA ligation dramatically affected estimated microRNA (miRNA) expression ( $A$ ), but the difference was comparatively small between libraries prepared with $10 \%$ or $15 \%$ PEG (B). Relative expression expressed as log fold-change for 15\% vs. 0\% PEG was significantly negatively correlated with miRNA GC-content, indicating an effect of RNA structure on ligation efficiency (C). Differences were smaller for $15 \%$ vs. 10\% PEG, and there was no significant correlation between $\log _{2}$ fold-change and GC-content (D). Addition of PEG increased library complexity with a smaller difference between 10\% and 15\% PEG and negligible differences between $1 \mathrm{~h}$ and 2 $\mathrm{h}$ ligation time (E). Expression is plotted as $\log _{2}$ of the counts per million miRNA reads (cpm).

(ATCC, Manassas, VA) was cultured in McCoy's 5A medium supplemented with 10\% FBS (both purchased from HyClone/GE Healthcare Europe GmbH, Freiburg, Germany). Total RNA was extracted using TRIzol (Ambion/Thermo Fisher Scientific, Waltham, MA) according to the manufacturer's instructions, and RNA concentrations were measured on a NanoDrop ND-1000 spectrophotometer (NanoDrop Technologies Inc., Wilmington, DE). The comparison with the miRQC study (12) was done using human brain reference RNA (Ambion/ Thermo Fisher Scientific) and universal human miRNA reference RNA (Stratagene/Agilent Technologies, Santa Clara, CA). Sequencing libraries were prepared with our protocol and with the NEBNext Multiplex Small RNA Library Prep Kit for Illumina (New England Biolabs, Ipswich, MA) according to the manufacturer's instructions.

A detailed protocol for the preparation of sequencing libraries is provided in the Supplementary Material. Briefly, $500 \mathrm{ng}$ of total RNA and 20 pmol of pre-adenylated 3 adapter were denatured for 2 min at $70^{\circ} \mathrm{C}$ before ligation for $1 \mathrm{~h}$ at $25^{\circ} \mathrm{C}$ in an $18 \mu$ reaction containing $200 \cup \mathrm{T} 4$ RNA ligase 2, truncated K227Q, 1× buffer for T4 RNA ligase, 15\% PEG MW 8000 (New England Biolabs), and 40 U RiboLock RNase inhibitor (Thermo Fisher Scientific, Waltham, MA). Next, 20 pmol of reverse transcription primer 
was added and annealed by incubation for $5 \mathrm{~min}$ at $75^{\circ} \mathrm{C}$, $15 \mathrm{~min}$ at $37^{\circ} \mathrm{C}$, and $15 \mathrm{~min}$ at $25^{\circ} \mathrm{C}$. For each reaction, 20 pmol of $5^{\prime}$ adapter was denatured separately for 2 min at $70^{\circ} \mathrm{C}$ and then ligated in $25 \mu$ reactions in the presence of $10 \cup$ T4 RNA ligase 1, 1× buffer for T4 RNA ligase, and $1 \mathrm{mM}$ ATP (New England Biolabs) for $1 \mathrm{~h}$ at $25^{\circ} \mathrm{C}$. For ligation tests, concentrations and incubation times were varied as specified in the "Results and discussion" section below. Reverse transcription was performed in 40 $\mu$ reactions with $200 \cup$ ProtoScript II reverse transcriptase in $1 \times$ reaction buffer, $10 \mathrm{mM}$ DTT, and $500 \mu \mathrm{M}$ dNTPs (New England Biolabs). Twenty microliters of cDNA was used for PCR amplification in $50 \mu$ reactions with $2 \times$ LongAmp Taq Master Mix (New England Biolabs) and $250 \mathrm{nM}$ each of indexed forward (8-nucleotide index; total size: 70 nucleotides) and reverse primers (6-nucleotide index; total size: 63 nucleotides). All oligonucleotides were purchased from Integrated DNA Technologies, Leuven, Belgium. Sequences are provided in Supplementary Table S1. These were adapted from Illumina oligonucleotide sequences.

For size selection by gel electrophoresis, PCR reactions were pooled and purified by extraction with phenolchloroform-isoamyl alcohol (25:24:1) before ethanol precipitation. After centrifugation and washing with $75 \%$ ethanol, the resulting pellets were resuspended in water and loading buffer before size separation on $1.5 \mathrm{~mm}$ thick, 6\% acrylamide gels (acrylamide/bis-acrylamide 19:1, Bio-Rad

\section{AccuCleave $\infty$

\section{Detecting CRISPR gene editing events just got QUICKR}

\author{
Introducing AccuCleave for use with \\ agarose gels
}

- Simple and accurate enzymatic digestion assay

- Reduce sequencing costs and times with efficient screening methods

\section{- Optimized Taq eliminates time consuming PCR cleanup}

Streamline your genetic editing workflows

at www.aati-us.com/AccuCleave
Laboratories, Hercules, CA) for 30 min at 180 V. Gels were stained with ethidium bromide and bands of $\sim 150-173 \mathrm{bp}$ (17-40 nucleotide inserts) were excised. Gel slices were fragmented before elution in $0.3 \mathrm{M} \mathrm{NaCl}$ for at least $2 \mathrm{~h}$ at room temperature. The eluate was purified on Spin-X columns (Corning, Tewksbury, MA) before ethanol precipitation. Final sequencing libraries were resuspended in water and quantified on a Qubit fluorometer using the dsDNA HS Assay Kit (both from Invitrogen/Thermo Fisher Scientific, Waltham, MA).

For size-selection tests with AMPure XP beads (Agentcourt/Beckman Coulter, Beverly, MA), purification was performed according to the manufacturer's instructions, but with $80 \%$ ethanol for washing. Dual selection was used with a bead:sample ratio of 1.1:1 for the first step to remove longer (bound) fragments; thus, the unbound material was transferred to new tubes for a second purification with a bead:sample ratio of 2.5:1 to remove shorter (unbound) DNA fragments such as adapter dimers. Library fragment sizes were analyzed with the High Sensitivity DNA Assay on a 2100 Bioanalyzer (Agilent Technologies, Santa Clara, CA).

Pooled libraries were sequenced on a NextSeq 500 with High Output v2 75 cycle kits for single-end reads or on a HiSeq 2500 with TruSeq Rapid SBS 200 cycle kits for paired-end reads (both sequencing systems and the associated kits used were obtained from Illumina, Inc., San Diego, CA). Sequences were demultiplexed with Picard (http://broadinstitute.github.io/picard/) and aligned using Novoalign (www.novocraft.com/products/novoalign/) with the following settings: - a TGGAATTCTCGGGTGCCAAGG -I 14 -h -1 -1 -t 90 -g 50 -x 15 -o SAM - o FullNW -r All 51 -e 51. miRNA expression was analyzed using custom Perl scripts and calculated as counts per million miRNA reads (cpm). The hg38 human genome assembly and miRBase v21 (13) were used for the ligation tests, while hg19 and miRBase v18 were used for comparison with the miRQC data. Statistical analyses were performed in $\mathrm{R}$.

\section{Results and discussion}

We have developed a rapid and scalable protocol for preparation of multiplexed small RNA sequencing libraries that combines the use of off-the-shelf reagents, early sample pooling, and dual indexing with sequencing on highthroughput sequencers to yield results fully comparable to commercially available kits at a considerably lower cost per sample. Our method is summarized schematically in Supplementary Figure S1 and described in detail in the protocol provided in the Supplementary Material.

Earlier studies showed that efficient ligation between oligonucleotide adapters and small RNAs is important to avoid introducing bias in sequencing libraries $(14,15)$. Ligation efficiency can be increased by adjusting reaction conditions, including incubation time and temperature, as well as by adding agents that affect molecular crowding, such as polyethylene glycol (PEG) (14). As is customary in most current protocols, we used truncated T4 RNA ligase 2 (K227Q mutant) together with a pre-adenylated $3{ }^{\prime}$ adapter in the first ligation step to increase efficiency 
A

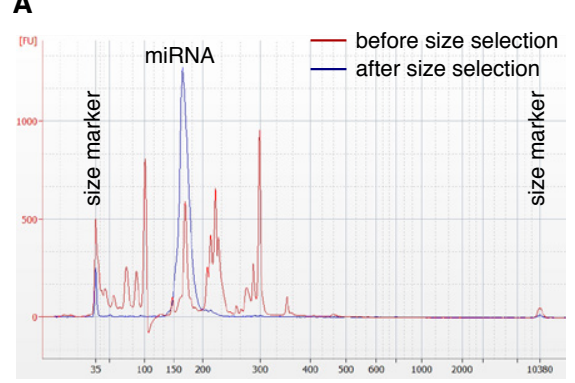

B

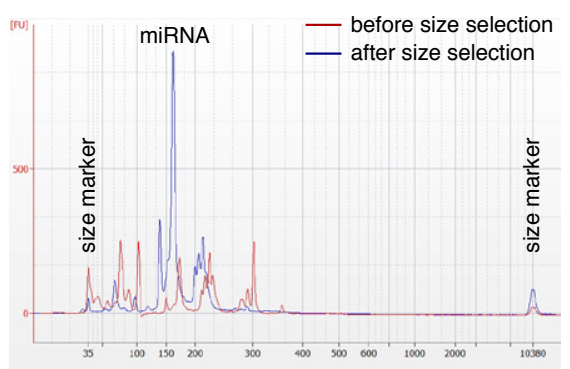

Figure 2. Gel purification is the preferred method for small RNA library size selection. DNA fragment size analysis showed that purification on 6\% acrylamide/acrylamide-bis (19:1) gels led to isolation of a narrow size range with efficient removal of adapter dimers (A), while bead-based size selection only partially removed larger DNA fragments and adapter dimers, even when using stringent bead:sample ratios (B). The library shown in (B) was subjected to 2 rounds of size selection, first with a bead:sample ratio of 1.1:1 to remove (bound) longer products followed by a ratio of 2.5:1 to remove shorter (unbound) material. Estimated DNA fragment sizes are shown in $\mathrm{bp}$ and fluorescence in arbitrary units (FU).

and reduce formation of by-products, such as circularized RNAs and RNA concatemers (16). RNA ligation reactions were conducted in the presence of varying concentrations of PEG, and the resulting samples were sequenced to evaluate the effects on library composition. Tests with $0 \%$, $10 \%$, or $15 \%$ (w/v) PEG MW 8000 and $1 \mathrm{~h}$ or $2 \mathrm{~h}$ incubation at $25^{\circ} \mathrm{C}$ are compared in Figure 1 (see Supplementary Tables S2 and S3 for quality control data and Supplementary Table S4 for miRNA expression). Ligation times can be extended further (e.g., to incubation overnight at $16^{\circ} \mathrm{C}$ ), but we limited the ligation time to allow us to prepare libraries and start sequencing within 2 days. We found that the addition of PEG had a marked effect on miRNA detection, with a smaller difference between libraries prepared with $10 \%$ or $15 \%$ PEG (Figure $1, A$ and $B)$. The difference between
A

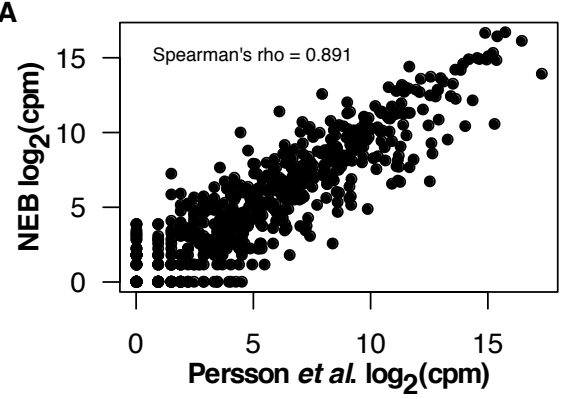

C

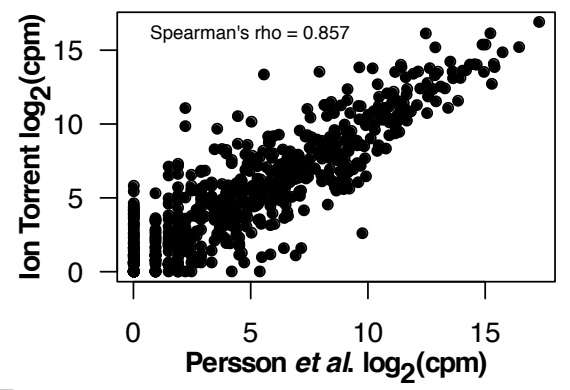

B

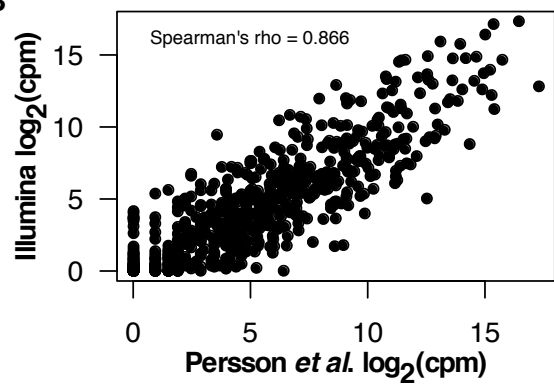

D

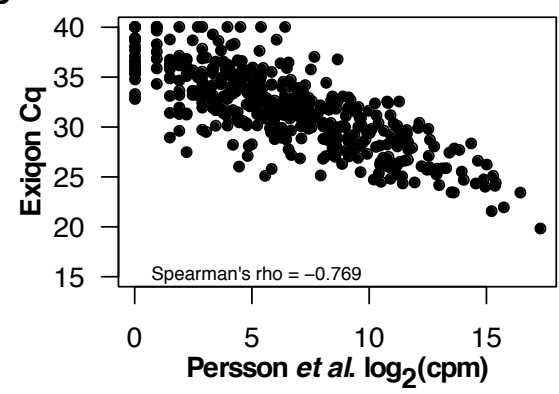

\begin{tabular}{lccccc}
\hline & Persson et al. & NEB & miRQC Illumina & miRQC lon Torrent & miRQC Exiqon \\
\hline Persson et al. & 1 & 0.891 & 0.866 & 0.857 & -0.769 \\
NEB & 0.891 & 1 & 0.891 & 0.821 & -0.749 \\
miRQC Illumina & 0.866 & 0.891 & 1 & 0.826 & -0.734 \\
miRQC lon Torrent & 0.857 & 0.821 & 0.826 & 1 & -0.840 \\
miRQC Exiqon & -0.769 & -0.749 & -0.734 & -0.840 & 1 \\
\hline
\end{tabular}

Figure 3. MicroRNA (miRNA) expression profiling performance compared between the current protocol and commercially available kits and platforms. Comparison between miRNA expression profiles for human brain reference RNA obtained using the method presented here and the NEBNext Multiplex Small RNA Library Prep Kit from Illumina (A). Also shown are comparisons with the IIlumina $(B)$ and IonTorrent $(C)$ sequence data, as well as the Exiqon real-time qRT-PCR data (D), from the miRQC study (12). Spearman rank correlations were similar between all methods, but somewhat higher among the sequencing-based platforms (E). Expression is shown as $\log _{2}$ of the counts per million miRNA reads (cpm) for sequence data and as $C_{q}$ values for real-time qRT-PCR data.

expression in $15 \%$ compared to $0 \%$ PEG, expressed as $\log _{2}$ fold-change, was significantly negatively correlated with the miRNA GC-content (Spearman's rho $=-0.12, P=0.008$ ), indicating an effect of RNA structure on ligation efficiency (Figure 1C). Expression differences were considerably smaller in libraries prepared in $15 \%$ compared to $10 \%$ PEG, and there was no significant correlation between $\log _{2}$ fold-change and GC-content (Figure 1D). Addition of PEG also increased library complexity substantially, with a smaller difference between 10\% and 15\% PEG and negligible differences between $1 \mathrm{~h}$ and $2 \mathrm{~h}$ ligation times (Figure 1E).

Sample preparation protocols for small RNA sequencing typically recommend libraries be gel-purified individually after PCR amplification to remove adapter dimers without inserts. If not removed, these can take up a significant amount of sequencing space. As an alternative, two rounds of size selection using AMPure XP beads to remove products that are larger or smaller than the intended size range is often suggested. Individual gel purification would be highly inconvenient when preparing large numbers of libraries, as well as being inexact due to laneto-lane variation in the excised gel slices, but bead-based size selection could be performed on a larger scale in 96-well plates for example. We decided to compare gel purification of a pool of barcoded libraries with two rounds of size selection using AMPure XP beads at several different bead:sample ratios. While gel purification produced a narrow size range with efficient removal of adapter dimers, bead-based size selection only partially removed larger DNA fragments and adapter dimers, even 
A

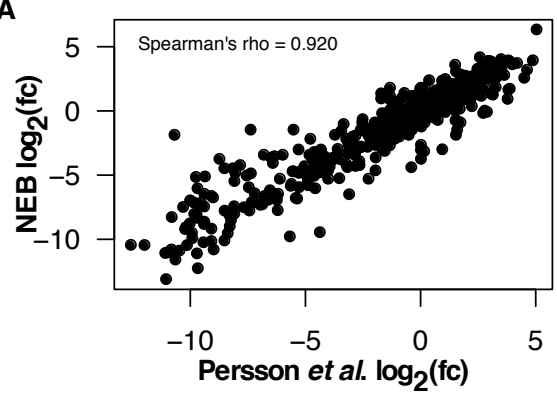

C

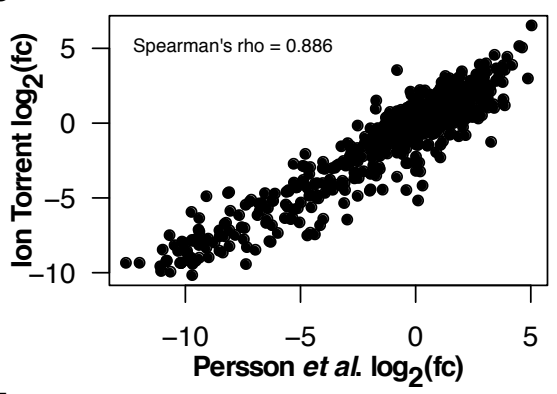

B

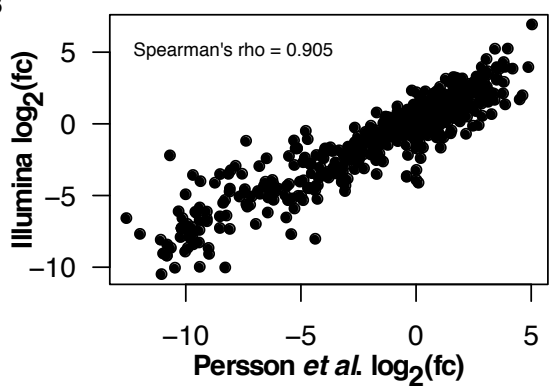

D

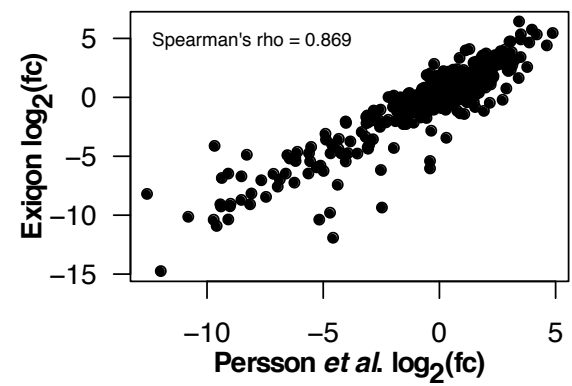

E

\begin{tabular}{lccccc}
\hline & Persson et al. & NEB & miRQC Illumina & miRQC Ion Torrent & miRQC Exiqon \\
\hline Persson et al. & 1 & 0.920 & 0.905 & 0.886 & 0.869 \\
NEB & 0.920 & 1 & 0.906 & 0.877 & 0.869 \\
miRQC Illumina & 0.905 & 0.906 & 1 & 0.948 & 0.942 \\
miRQC lon Torrent & 0.886 & 0.877 & 0.948 & 1 & 0.945 \\
miRQC Exiqon & 0.869 & 0.869 & 0.942 & 0.945 & 1 \\
\hline
\end{tabular}

Figure 4. Differential expression of microRNAs (miRNAs) between samples is well-captured by the current method. Differential expression between human brain reference RNA and universal human miRNA reference RNA expressed as $\log _{2}$ (fold-change, fc) compared between data obtained using the method presented here and the NEBNext Multiplex Small RNA Library Prep Kit for Illumina (A). Also shown are comparisons with the Illumina (B) and IonTorrent (C) sequence data, as well as the Exiqon real-time qRT-PCR data (D), from the miRQC study (12). Spearman rank correlations were similar between all methods, but somewhat higher among data from the miRQC study (E). This might be caused, for example, by differences in the RNA pools or sequencing depth.

when using stringent bead:sample ratios (Figure 2, A and B). The library shown in Figure 2B was subjected to two rounds of size selection, first with a bead:sample ratio of 1.1:1 to remove (bound) longer products followed by a ratio of $2.5: 1$ to remove shorter (unbound) material. Increasing stringency further (1.2-1.3:1 followed by 1.6-1.8:1) did not solve the issue and led to the loss of material in the miRNA size range. We concluded that AMPure $\mathrm{XP}$ beads could not efficiently isolate the desired size range and decided to use gel purification for a common pool of all samples to minimize hands-on time and sample-to-sample variation. The pool was made after amplification with indexed PCR primers and purified by phenol-chloroform extraction

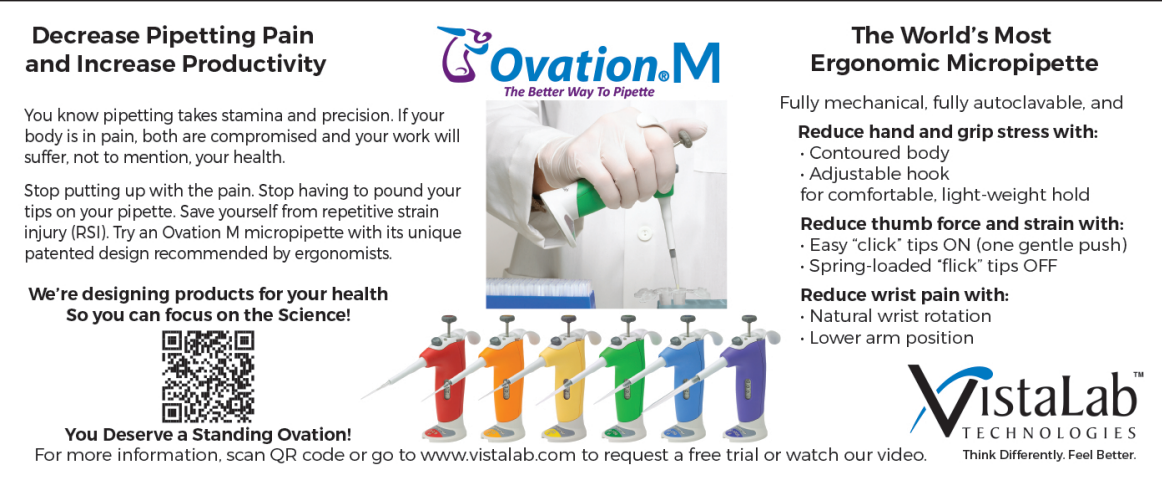

and ethanol precipitation before gel electrophoresis. Optionally, a PCR clean-up kit could be used to purify and concentrate sequencing libraries before size selection.

A potential concern for pooling $\mathrm{PCR}$ reactions prior to purification is contamination between libraries, which could affect biological and clinical data interpretation. Such contamination is likely to result in identification within a read of pairs of forward and reverse primer indices that were not used together during sample preparation. We therefore analyzed the number of used and unused index combinations that could be detected during de-multiplexing with Picard (http:// broadinstitute.github.io/picard/). The data consisted of a library pool where all 4 forward and 24 reverse primers were used (forward primers 1 and 3 with reverse primers 1-12 and forward primers 2 and 4 with reverse primers 13-24, for a total of 48 unique combinations). The average percentage of unused reverse primer indices detected in reads containing our 4 different forward primers was as low as $0.02 \%$ among reads with perfect matches to the index sequences and $0.08 \%$ when allowing for 1 mismatch. Since a quality score of 30 (a commonly used cut-off for Illumina data) corresponds to one erroneous base call in $1000 \mathrm{bp}$, or an error rate of $0.6 \%$ for a 6-bp index sequence, this suggests that sequencing errors are a more likely explanation than contamination between libraries. Furthermore, when detected, the unused reverse primer indices had a significantly lower mean base quality score than used indices (e.g., 13.5 versus 30.9 and 21.1 versus 30.8 for partners of 2 different forward primer indices, $P<2.2 \times 10^{-16}$ for both according to Student's $t$-test). Taken together, these results indicate that no contamination between libraries could be detected after pooling of PCR reactions prior to purification.

Finally, we evaluated the performance of our experimental protocol by sequencing the human brain and universal reference RNAs that were used in the miRQC study for the comparison of platforms for miRNA expression analysis (12). Sequencing libraries were prepared from these two 
samples using the method presented here, as well as with a commercially available kit (NEBNext Multiplex Small RNA Library Prep Kit for Illumina), and the resulting miRNA expression profiles were compared with publicly available data from the miRQC study by Mestdagh et al. (12). Quality control data are presented in Supplementary Tables S2 and S3 and miRNA expression data in Supplementary Table S5. Comparisons with absolute miRNA expression in $\log _{2}$-transformed counts per million (cpm) miRNA reads for two different sequencing platforms (Illumina and Ion Torrent), as well as one real-time qRT-PCR-based method, are shown in Figure 3. Spearman rank correlations were similar between all methods, but somewhat higher among the sequencing-based platforms. The range of correlations (Spearman's rho $=0.8-0.9$ ) indicates that our protocol for preparation of sequencing libraries performs favorably when compared with the commercially available kits, especially considering that the work was performed in another lab by other people and several years later (Figure $3 E)$. The results were similar for the universal human reference RNAs (data not shown). Figure 4 summarizes the comparison for differential expression of miRNAs, shown as $\log _{2}$ fold-change for brain versus universal human reference RNA. Again, our results correlate well with those obtained from commercial platforms in the miRQC study.

Here, we developed a rapid, economical, and scalable protocol for the preparation of multiplexed small RNA sequencing libraries. Our method is compatible with dual indexing using standard Illumina high-throughput sequencing kits. For example, library pools containing all 96 index combinations could be sequenced on the NextSeq (High Output kit) or HiSeq (Rapid Run Mode) to yield up to 4 million or 3 million reads per sample, respectively. A strategy for increasing sample throughput for SOLiD sequencing of small RNAs by dual indexing was suggested by Tu et al. (11). However, this method was based on the addition of one set of indices during adapter ligation, an approach that has been shown to introduce bias in miRNA detection (17-18). Size selection was also performed by gel purification of individual libraries, limiting throughput.

Our optimization experiments showed the importance of increasing adapter ligation efficiency by addition of PEG to minimize bias in library composition. We have also demonstrated that it is possible to pool PCR-amplified libraries before purification in order to allow size selection on acrylamide gels, which is preferable to bead-based methods for isolation of a narrow size range. Finally, by comparing our data with data from the $\mathrm{MiRQC}$ study for the validation of miRNA expression analysis platforms (12), we have confirmed our method captures both absolute and differential expression as well as the commercially available alternatives. It is noteworthy that, although the results from all of these platforms are fairly concordant, the correlations are not perfect, indicating caution must be used when combining small RNA expression data generated using different experimental protocols. We are currently using this protocol for all of our miRNA expression analysis projects, both low- and high-throughput $(\geq 200$ libraries prepared manually in $<2$ days by 1 person), and believe that it could also be useful for other researchers performing small RNA sequencing on a regular basis.

\section{Author contributions}

H.P. and C.R. conceived the study. H.P. designed the study. R.S. contributed to the design of the study. H.P. and A.P. performed the experiments. H.P. analyzed the data. H.P. and C.R. wrote the manuscript. All authors read and approved the final version of the manuscript.

\section{Acknowledgments}

The study was supported by grants from the Berta Kamprad Foundation and the Swedish Cancer Society (to C.R.) and from the Bertil Andersson fund (to H.P.).

\section{Competing interests}

The authors declare no competing interests.
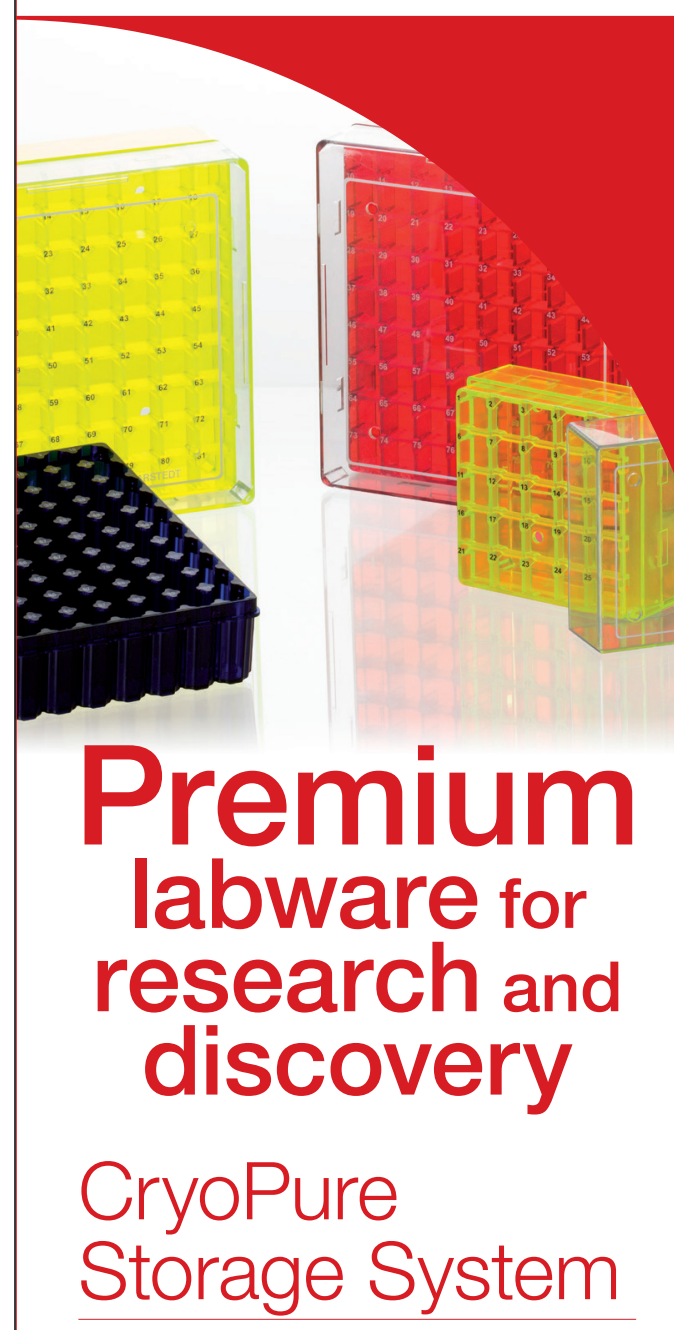

- Certified tubes, racks and boxes for cryogenic storage

- Volumes from 1.2 to $5.0 \mathrm{ml}$

- Skirted bases and QuickSeal caps for ergonomic handling

- 6 cap and insert colors for up to 36 color coding options
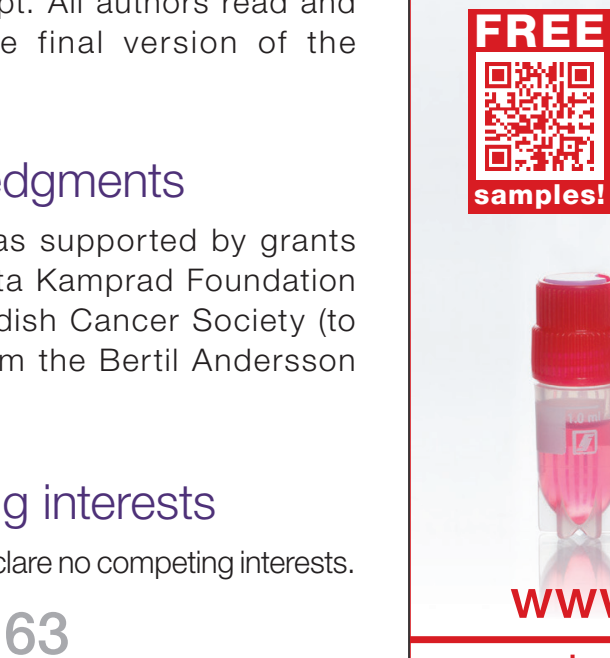


\section{References}

1. Bartel, D.P. 2004. MicroRNAs: genomics, biogenesis, mechanism, and function. Cell 116:281-297.

2. Jonas, S. and E. Izaurralde. 2015. Towards a molecular understanding of microRNAmediated gene silencing. Nat. Rev. Genet. 16:421-433

3. Kinet, V., J. Halkein, E. Dirkx, and L.J. Windt. 2013. Cardiovascular extracellular microRNAs: emerging diagnostic markers and mechanisms of cell-to-cell RNA communication. Front. Genet. 4:214.

4. Jopling, C.L., M. Yi, A.M. Lancaster, S.M. Lemon, and P. Sarnow. 2005. Modulation of hepatitis $C$ virus RNA abundance by a liverspecific MicroRNA. Science 309:1577-1581.
5. Adams, B.D., A.L. Kasinski, and F.J. Slack. 2014. Aberrant regulation and function of microRNAs in cancer. Curr. Biol. 24:R762R776.

6. Lagos-Quintana, M., R. Rauhut, W. Lendeckel, and T. Tuschl. 2001. Identification of Novel Genes Coding for Small Expressed RNAs. Science 294:853-858.

7. Lau, N.C., L.P. Lim, E.G. Weinstein, and D.P. Bartel. 2001. An abundant class of tiny RNAs with probable regulatory roles in Caenorhabditis elegans. Science 294:858-862

8. Lee, R.C. and V. Ambros. 2001. An extensive class of small RNAs in Caenorhabditis elegans. Science 294:862-864.

9. Persson, H., A. Kvist, N. Rego, J. Staaf, J. Vallon-Christersson, L. Luts, N. Loman, G.

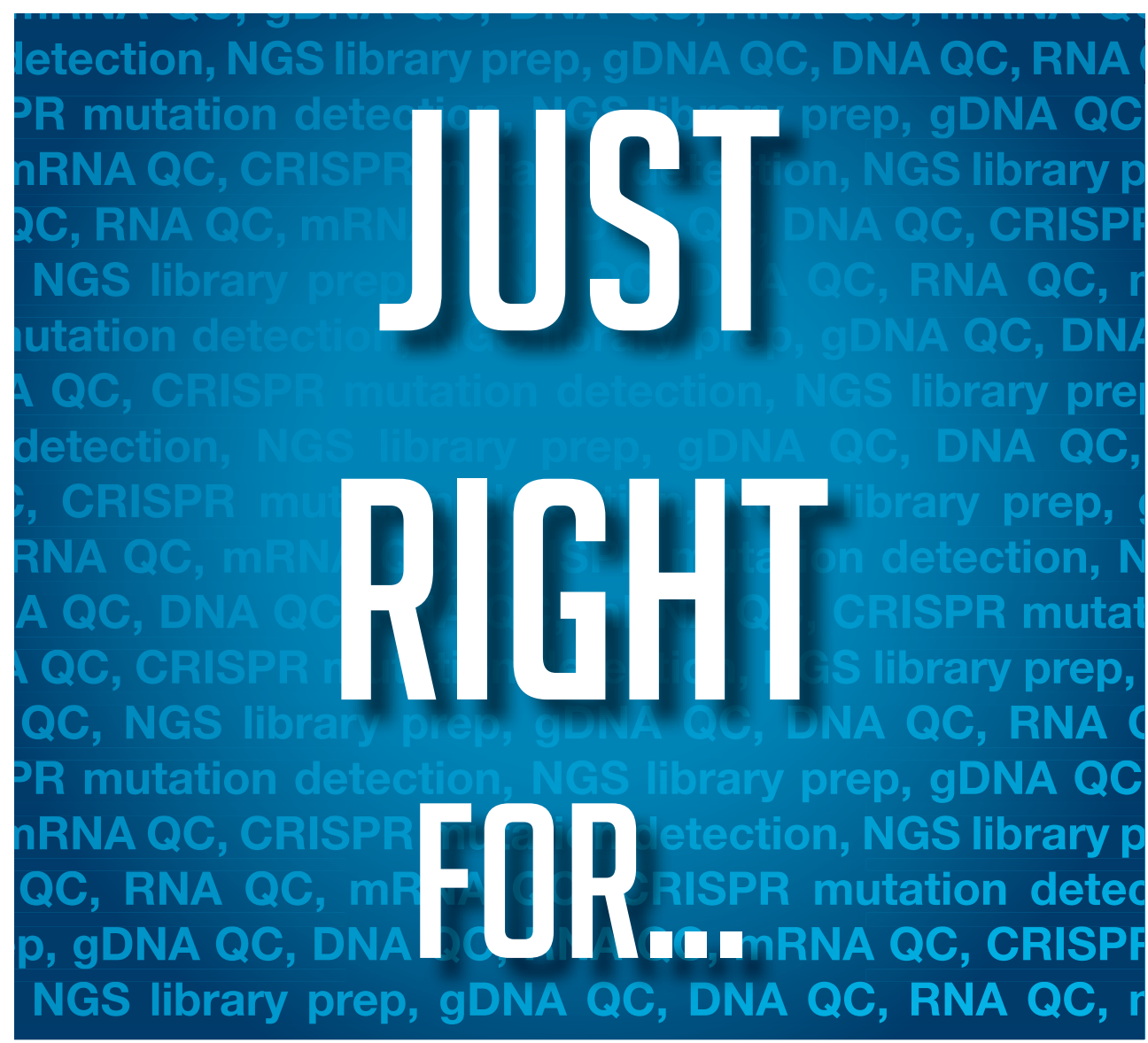

STREAMLINE GENOMIC WORKFLOWS WITH AUTOMATED NUCLEIC ACID QC

The Fragment Analyzer ${ }^{\mathrm{TM}}$ is the premier instrument for automating QC steps in genomics workflow, from raw nucleic acids to final library validation.

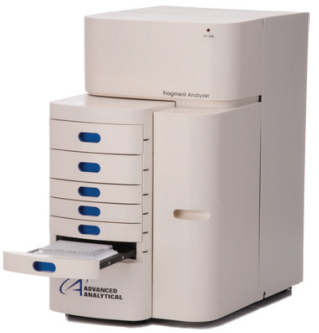

Jonsson, et al. 2011. Identification of new microRNAs in paired normal and tumor breast tissue suggests a dual role for the ERBB2/ Her2 gene. Cancer Res. 71:78-86.

10. Boele, J., H. Persson, J.W. Shin, Y. Ishizu, I.S. Newie, R. Sokilde, S.M. Hawkins, C. Coarfa, et al. 2014. PAPD5-mediated 3 adenylation and subsequent degradation of miR-21 is disrupted in proliferative disease. Proc. Natl. Acad. Sci. USA 111:11467-11472.

11. Tu, J., Q. Ge, S. Wang, L. Wang, B. Sun, Q. Yang, Y. Bai, and Z. Lu. 2012. Pair-barcode highthroughput sequencing for large-scale multiplexed sample analysis. BMC Genomics 13:43.

12. Mestdagh, P., N. Hartmann, L. Baeriswyl, D. Andreasen, N. Bernard, C. Chen, D. Cheo, P. D'Andrade, et al. 2014. Evaluation of quantitative miRNA expression platforms in the microRNA quality control (miRQC) study. Nat. Methods 11:809-815.

13. Kozomara, A. and S. Griffiths-Jones. 2011 miRBase: integrating microRNA annotation and deep-sequencing data. Nucleic Acids Res. 39:D152-D157.

14. Munafó, D.B. and G.B. Robb. 2010. Optimization of enzymatic reaction conditions for generating representative pools of cDNA from small RNA. RNA 16:2537-2552.

15. Hafner, M., N. Renwick, M. Brown, A. Mihailovic, D. Holoch, C. Lin, J.T. Pena J.D. Nusbaum, et al. 2011. RNA-ligasedependent biases in miRNA representation in deep-sequenced small RNA cDNA libraries. RNA 17:1697-1712.

16. Viollet, S., R.T. Fuchs, D.B. Munafo, F. Zhuang, and G.B. Robb. 2011. T4 RNA ligase 2 truncated active site mutants: improved tools for RNA analysis. BMC Biotechnol. 11:72

17. Alon, S., F. Vigneault, S. Eminaga, D.C. Christodoulou, J.G. Seidman, G.M. Church, and E. Eisenberg. 2011. Barcoding bias in high-throughput multiplex sequencing of miRNA. Genome Res. 21:1506-1511.

18. Van Nieuwerburgh, F., S. Soetaert, K. Podshivalova, E. Ay-Lin Wang, L. Schaffer, D. Deforce, D.R. Salomon, S.R. Head, and P. Ordoukhanian. 2011. Quantitative bias in Illumina TruSeq and a novel post amplification barcoding strategy for multiplexed DNA and small RNA deep sequencing. PLoS One 6:e26969.

Received 29 March 2017; accepted 20 June 2017

Address correspondence to Helena Persson, Department of Clinical Sciences Lund, Oncology and Pathology, Lund University Cancer Center, Scheelevägen 2, SE-223 81, Lund, Sweden. E-mail: helena.persson@med.lu.se

To purchase reprints of this article, contact:

biotechniques@fosterprinting.com 